\title{
MHD Stability Analysis Of Type II ELMs In ASDEX Upgrade
}

\author{
S. Saarelma ${ }^{1}$, S. Günter ${ }^{2}$, T. Kiviniemi ${ }^{1}$, T. Kurki-Suonio ${ }^{1}$ and ASDEX \\ Upgrade TEAM ${ }^{2}$ \\ ${ }^{1}$ Helsinki Univ. of Tech., Euratom-TEKES Association, FIN-02015 HUT, Finland \\ 2 Max-Planck-Institut für Plasmaphysik, D-85748, Garching, Germany
}

\begin{abstract}
An MHD stability analysis of the plasmas with type II ELMs is presented. The stability properties in type II ELMy plasmas (high- $q_{95}$, high $\delta$ ) are found to significantly differ from those of the type I ELMy plasmas. The characteristics of the type II ELM cycle due to these stability properties are described.
\end{abstract}

\section{Introduction}

The type II or grassy ELMs show potential for a steady-state operation of a tokamak with good confinement and efficient impurity exhaust while not damaging the divertor plates. The energy lost in a single type II ELM is significantly lower than in a type I ELM. Respectively, the ELM frequency of type II ELMs is much higher.

The ELM phenomenon has several explanations [1]. Here the model presented for type I ELMs by Connor et al. [2] is studied for type II ELMs in ASDEX Upgrade. The significant differences in the characteristics of the instabilities leads to different plasma behavior, and consequently, also to different ELM cycle.

\section{Plasma Conditions for Type II ELMs}

On ASDEX Upgrade, type II or grassy ELMs have been observed only if density is high $\left(\overline{n_{e}} / \overline{n_{G W}} \geq 0.85\right), q$ at the edge is high $\left(q_{95} \geq 4.2\right)$, and triangularity is high $(\delta=0.40)$ [3]. The edge profiles of the temperature and the density are comparable to the type I ELMy plasmas and significantly steeper than in type III ELMy plasmas. High $q_{95}$ and high triangularity are associated with grassy ELMs also at JT-60U [4]. In addition to high triangularity and high safety factor at the edge, type II ELMs were observed also when plasma approached double-null configuration [3].

\section{Equilibrium Reconstruction}

The bootstrap current plays an important role in the stability of the edge plasma. It is proportional to the pressure gradient, and consequently, due to the steep pressure gradient of the H-mode plasmas, the bootstrap current dominates the inductively driven current near the edge. The bootstrap current was included in the equilibrium calculation using an analytical formula for the flux averaged parallel bootstrap current $\langle\mathbf{j} \cdot \mathbf{B}\rangle$ given by Sauter et al. [5]. The inductively driven current was assumed to dominate in the core, and its profile was chosen to fit with the $q$-profile information in the core. The total current profile was therefore a superposition of the inductively driven current and the bootstrap current. This allowed studying realistically the stability effect of the bootstrap current that is dependent on the pressure gradient.

The preliminary studies with an guiding-centre orbit-following simulations suggest that at the very edge, in addition to the bootstrap current there is also a significant current contribution from the ion orbit losses. However, this current is not yet included in the equilibrium calculation. 
The pressure profile is not significantly different for type I and type II ELMy regions [3]. Therefore, it is kept fixed in analyzing the differences of the stability properties of these plasmas. Figure 1 shows the shapes of the pressure and $q$ profiles used in the equilibrium reconstruction.
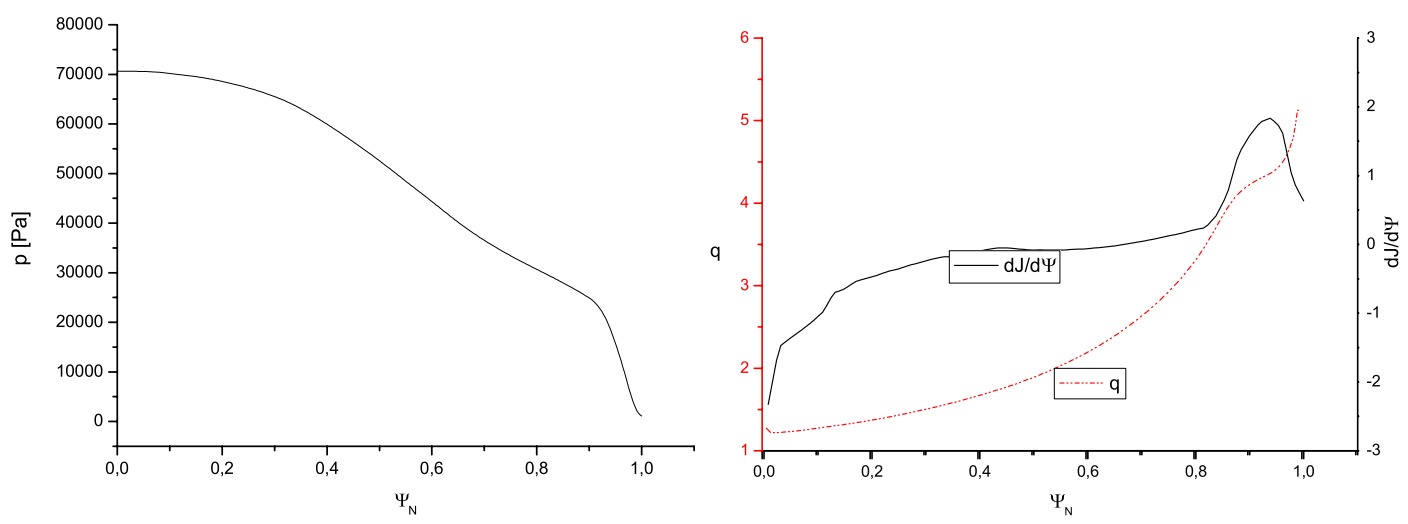

Figure 1: The smoothed pressure profile used in the equilibrium reconstruction as well as the resulting poloidal current density and the $q$-profile. The bootstrap current affects strongly the current profile at the H-mode barrier near the edge.

\section{Stability Analysis}

The ideal low-n stability of the plasma was investigated using the GATO [6] code. In the stability analysis, the plasma triangularity and edge safety factor were varied. Type I ELM plasma was characterized with low triangularity and low $q_{95}$. Type II ELM plasma was created by increasing the triangularity and $q_{95}$. It was found that when the triangularity and $q$-profile are increased, the plasma edge stability against current driven peeling modes increases. Here, the increased stability means that higher bootstrap current fraction is needed to drive the plasma peeling mode unstable. Also for the unstable modes with the same bootstrap current, the growth rate of the instability is lower.

In addition to the stabilizing effect, the eigenfunction of the unstable mode becomes more localized. This is shown in Fig. 2. It must be noted here that neither of the factors $\left(\delta\right.$ or $\left.q_{95}\right)$ is sufficient alone to cause the localization of the instability, but both are required.

The effect of the double-null configuration was investigated by keeping all other parameters as constant as possible and moving the second separatrix close to the plasma (a few $\mathrm{mm}$ at the equatorial plane). It was found that, while the eigenfunction of the instability does not become more localized as happened with high triangularity and high $q$ at the edge, the plasma is more stable than in single null configuration.

In addition to low-n instabilities, also the high-n ballooning stability of the plasma was investigated. No significant difference was found between type I and type II plasma conditions. The experimental pressure gradient near the edge was close to the stability limit for both plasmas.

It can be assumed that the non-linear behavior of the instability is similar for wide and narrow instabilities, and the width of the increased transport is dependent on the width of the unstable part of the plasma. Then the localization of the instability indicates that the amount of plasma that is removed by the peeling mode from the edge also follows the width of the eigenfunction. Thus, less plasma is removed in an instability with type II ELM conditions (high $\delta$, high $q_{95}$ ) than in type I ELM conditions (low $\delta$, low $q_{95}$ ). 

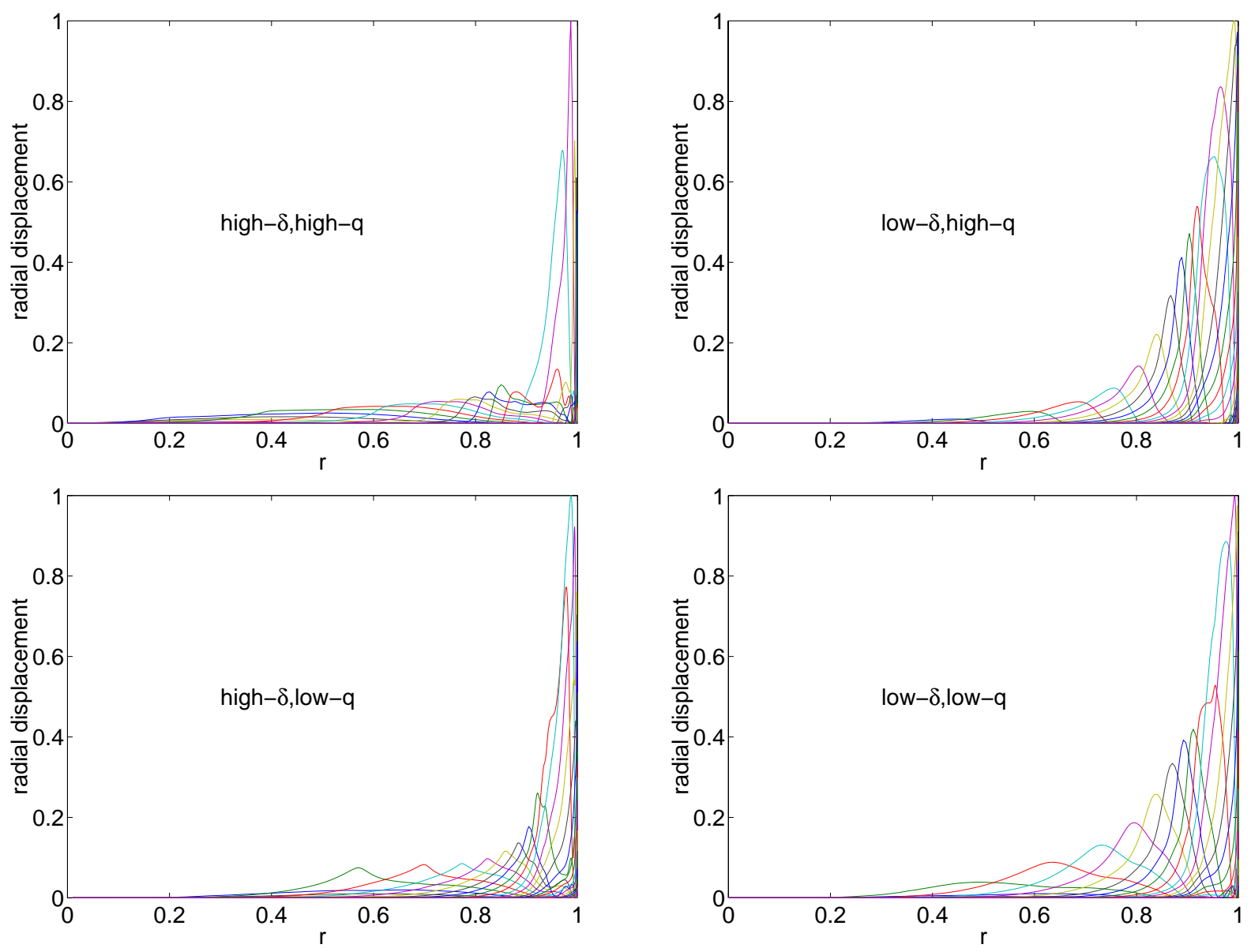

Figure 2: Fourier analysis of the eigenfunctions of the radial displacement of the $n=3$ peeling mode for different $q_{95}-\delta$ combinations.

There is also another mechanism that explains, why in type II ELM conditions less plasma is lost. Higher edge current is needed to destabilize the plasma with high triangularity and $q$ at the edge. However, the pressure gradient is comparable to type I conditions because it is limited by the ballooning modes. Consequently, when the ELM crash happens, the type II ELM plasma is already close to the steady state bootstrap current, while the bootstrap current in type I ELM plasma is far from saturation. The steady state is defined as the maximum value where bootstrap current would rise, if there were no instabilities. When the instability starts, it removes plasma at the edge and the pressure gradient starts to decrease. The steady state bootstrap current that is proportional to the pressure gradient decreases with the pressure gradient. If the edge current is still far from the steady state condition, it does not immediately start to decrease as the pressure gradient relaxes due to the increased transport, thus keeping the plasma edge unstable longer.

In type II ELM conditions, this limit is reached earlier than in type I ELM conditions. This means that the pressure gradient decreases less and also less plasma is lost in the instability. The new stable state is also close to the state, where plasma was just before the instability was triggered.

Since less plasma is lost at the edge, also the decrease in the edge current density is smaller. Consequently, the time to build up the current after an ELM-crash for the next 
instability would be shorter for type II ELMs. The density is found to be higher in type II ELMy plasmas than in type I ELMs. The higher density makes the current diffusion faster, thus further shortens the time between two ELMs.

All these effects lead to a higher ELM frequency and smaller energy loss during each ELM in type II ELMy conditions than in type I ELM conditions. The differences between the type I and type II ELM cycles are shown in Fig. 3.
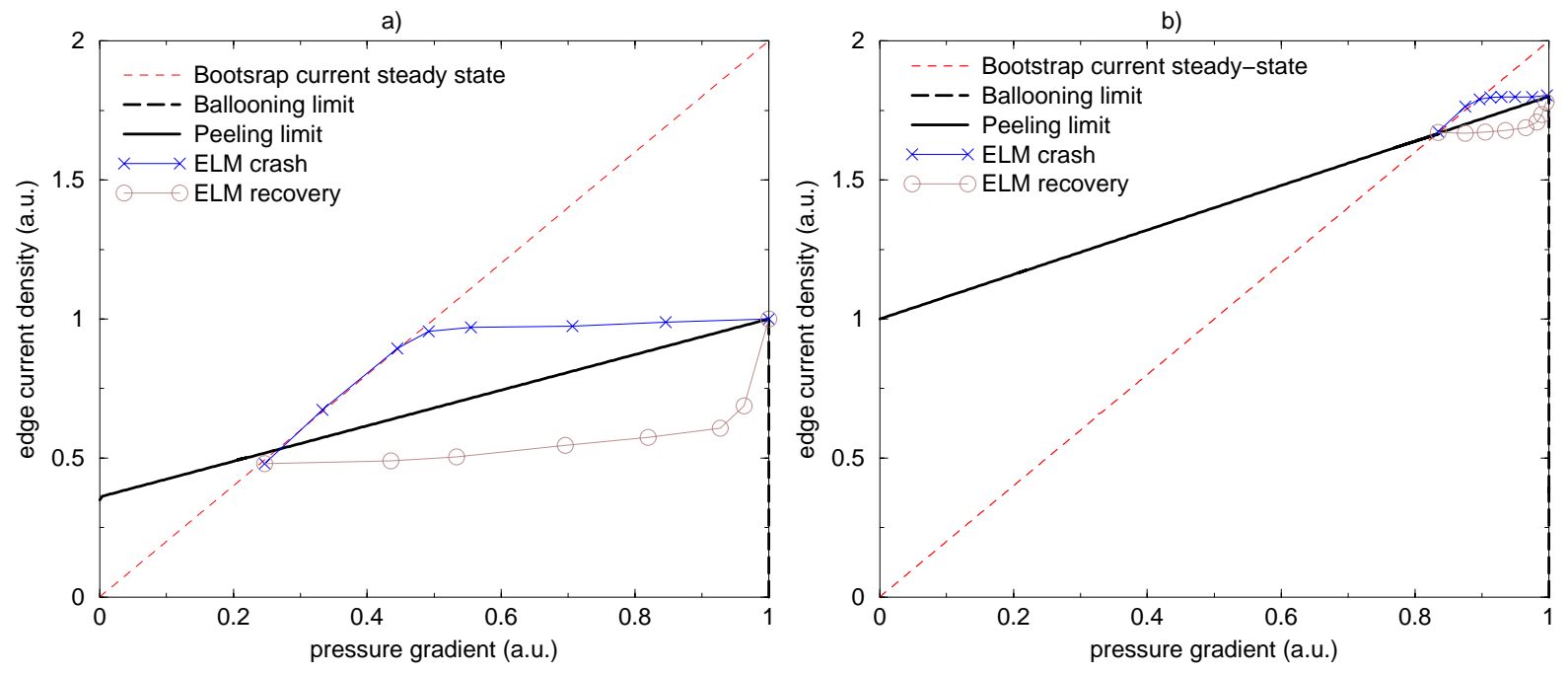

Figure 3: a) Type I ELM cycle, b) Type II ELM cycle.

\section{Conclusions and Discussion}

In ASDEX Upgrade type II ELMs are observed in plasmas with high triangularity and high safety factor at the edge. In these conditions, the numerical stability analysis shows the plasma to be more stable against peeling modes and have more localized mode structure than in comparable type I ELMy plasma. In addition the higher density makes the current diffusion faster. All this leads to more frequent but less intense ELM bursts. To make an even more careful analysis of the edge plasma, also the ion orbit loss current must be included in the equilibrium.

\section{References}

[1] J.W. Connor, Plasma Physics and Controlled Fusion, 40 (1998), 191-213

[2] J.W. Connor, R.J. Hastie, H.R. Wilson, Physics of Plasmas, 5 (1998), 2687-2700

[3] J. Stober invited talk (12) in this conference

[4] Y. Kamada et al. Plasma Phys. and Contr. Fusion, 42 (2000), A247-A253

[5] O. Sauter, C. Angioni, Y.R. Lin-Liu, Physics of Plasmas, 6 (1999), 2834-3839

[6] L. C. Bernard, F. J. Helton, R. W. Moore, Comp. Phys. Comm. 24 (1981) 377 